\title{
CONDICIONES DE EDUCABILIDAD Y RESULTADOS ESCOLARES EN BARRIOS VULNERABLES DE LA CIUDAD DE BAHÍA BLANCA, ARGENTINA. ${ }^{1}$
}

\section{EDUCABILITY CONDITIONS AND SCHOOL RESULTS IN VULNERABLE NEIGHBORHOODS OF THE CITY OF BAHÍA BLANCA, ARGENTINA.}

\author{
María Marta Formichella \\ Instituto de Investigaciones Económicas y Sociales del Sur, \\ IIESS (UNS-CONICET) \\ Departamento de Economía, Universidad Nacional del Sur (UNS), Ar- \\ gentina \\ mformichella@iiess-conicet.gob.ar \\ Natalia Krüger \\ Instituto de Investigaciones Económicas y Sociales del Sur, \\ IIESS (UNS-CONICET) \\ Departamento de Economía, Universidad Nacional del Sur (UNS), Ar- \\ gentina \\ natalia.kruger@uns.edu.ar
}

\section{Cómo citar / citation}

Formichella, M. M. y Krüger, N. (2019) "Condiciones de educabilidad y resultados escolares en barrios vulnerables de la ciudad de Bahía Blanca, Argentina”. OBETS. Revista de Ciencias Sociales, 14(1): 89-118. doi: 10.14198/OBETS2019.14.1.03

\footnotetext{
${ }^{1}$ El presente trabajo fue realizado en el marco del Proyecto de Desarrollo Tecnológico y Social (PDTS) 392, financiado por el CIN y el CONICET, y del Proyecto de Grupos de Investigación (PGI) 24/ZE33 "Equidad ducativa: Segmentación escolar en la localidad de Bahía Blanca", financiado por la SCyT, UNS.
} 


\title{
Resumen
}

Este trabajo analiza los datos de una encuesta de hogares realizada en tres barrios vulnerables de la ciudad de Bahía Blanca, Argentina, con el objetivo de evaluar la influencia del nivel socioeconómico y otros factores personales, familiares y escolares, sobre los resultados educativos (como asistencia y repitencia). Se realiza un análisis de conglomerados y de interpretación estadística-descriptiva. Se encuentra que, incluso dentro de esta población vulnerable, las desigualdades socioeconómicas iniciales parecen traducirse en desigualdades educativas. Más interesante es que se detectan posibles factores de mediación que permiten a algunos de los niños más desfavorecidos presentar mejores trayectorias educativas, y viceversa.

Palabras clave: equidad; inclusión; educación básica; análisis de conglomerados.

\begin{abstract}
This paper analyzes data from a household survey conducted in three vulnerable neighborhoods in the city of Bahía Blanca, Argentina, with the aim of evaluating the influence of socioeconomic status and a wider array of personal, family and school factors on educational results, such as attendance and repetition. When performing a cluster analysis and an interpretation of descriptive statistics, the results indicated that, even within this vulnerable population, initial socioeconomic inequalities seem to be translating into educational inequalities. More interestingly, possible mediating factors, that allow some of the most disadvantaged children to present better educational trajectories, and vice versa, are identified.

Keywords: equity; inclusion; basic education; cluster analysis.

\section{Extended Abstract}

Being education an essential capability (Sen, 1999) -a fundamental freedom that allows people to choose the lifestyle they wish to have- the widely documented fact that socioeconomic origin strongly influences school trajectories implies that education may reproduce social injustice. However, as Longás Mayayo et al. (2016) have emphasized, other factors belonging to different levels of analysis are at stake in a complex phenomenon such as school success. It is of interest, then, to identify those elements that may favor the development of desirable educational results even in vulnerable environments. This would enhance the possibility of designing policies, complementary to the unavoidable action in the socioeconomic sphere, to increase social and educational equity.
\end{abstract}


With this motivation, our research team has implemented the Technological and Social Development Project (TSDP) "Designing strategies to improve the educational opportunities of the vulnerable population of Bahia Blanca through the NGO Network of Volunteers". The project, led by a multidisciplinary group of researchers from three public universities, seeks to develop a strategic plan for the NGO aimed at strengthening its interventions in three poor neighborhoods of Bahía Blanca (Buenos Aires, Argentina). The NGO's work is particularly focused on promoting children and teenagers' right to education, in a context were poverty traps are evident. The combination of low levels of education and work-related problems among adults, as well as deficient housing and health conditions, reduces the chances of achieving high quality educational results for the children.

As a part of this project, this paper aims to make a contribution through the following specific objectives: i) although the entire population considered is vulnerable compared to the rest of the city, we seek to know whether it presents internal heterogeneity regarding the socioeconomic conditions of the households, so that differentiated groups may be identified; ii) then, we compare indicators of educational inclusion between and within these groups, in order to evaluate whether socioeconomic status helps explain the inequalities registered within these disadvantaged social sectors; iii) we analyze whether some children have unexpected positive or negative educational results relative to their group, and try to identify specific personal, family and contextual factors that may be moderating the impact of socioeconomic status.

In order to do this, we employed a primary source of information: data from a household survey carried out during the project, in April 2016. A household and an individual questionnaire were designed by the research team. The first one collected general information about the household`s members and living conditions (demographic composition, sources of income, household assets, housing conditions, parents`opinions on education, availability of resources and conditions for schooling, characteristics of the neighborhood, among others). The individual questionnaire was applied to children and young people from 3 to 18 years of age, and was aimed at characterizing their current educational situation, their educational trajectory, their perceptions and expectations regarding their school and education in general, etc.). A probabilistic sampling design was carried out and, finally, 381 households and 1459 people were surveyed who, after applying the corresponding sampling weight, represented a total of 1077 households and 4125 people. 
The methodology was quantitative and involved multivariate analysis and the interpretation of different descriptive statistics. First, we performed a cluster analysis of the households based on a selection of socioeconomic status indicators: educational level of the adults in the household, total monthly income, and unsatisfied basic needs (different housing conditions and employment). We reached an optimal partition of two clusters of households, characterized them in terms of a wider set of socioeconomic variables, and found that they present significantly different socioeconomic conditions.

Then, we compared the situation of the children and teenagers belonging to each group in terms of school attendance, repetition and truancy, dividing the population according to their school age: preschool, primary and high school. We found that the cluster with better socioeconomic conditions also presents better educational indicators: attendance to preschool is significantly higher; repetition and truancy at the primary level is lower; and attendance among the children in secondary school age is much higher, while repetition and absenteeism are clearly lower. In sum, even within these poor neighborhoods, initial socioeconomic inequalities seem to be translating into educational inequalities.

Going beyond these average characterizations of the clusters, we then proceeded to focus on two specific groups: on the one hand, children who belong to the poorest cluster and yet present the best possible educational result (attendance to the modal grade) and those who belong to the relatively advantaged cluster and still present the worst possible results (dropout or attendance with more than one repetition). We compared both groups considering a more complete set of variables, and identified some interesting results: the group with above-average educational results presents a higher proportion of female-headed households or with nuclear families; a higher proportion of children and teenagers who often play sports, go to the cinema or read; a higher availability of home educational resources; a lower amount of time devoted to household chores by the children and a higher amount of time dedicated to studying; and a higher level of parental interest in school homework or escorting children to school. As for the school characteristics, the use of computers, the attendance to longer schooldays and the supply of a meal at school are more widespread among the schools attended by the children in the first clusters. In sum, although these analyses are modest and should be replicated and extended, many other factors seem to be interacting with socioeconomic background, allowing some of the most disadvantaged children to present better educational trajectories, and vice versa. 
These findings represent a useful input for extending the agenda of the research team, perhaps applying qualitative methods to further analyze the role of possible mediating. Moreover, results may aid the improvement of intervention strategies by the NGO and the work of local policymakers, by drawing attention to the urgent action required in these vulnerable neighborhoods, both in the socioeconomic and educational spheres.

\section{Introducción}

En la literatura económica existe consenso acerca de que la educación genera efectos benéficos en el desarrollo social, pero al mismo tiempo se resalta la importancia de que sea impartida con equidad (Formichella, 2010; Krüger, 2016). Sen (1999) define al desarrollo como un proceso de expansión de las capacidades de los individuos, a las cuales precisa como las libertades fundamentales que permiten a las personas optar por aquel estilo de vida que desean tener. Así, explica que la educación es una capacidad esencial por la multiplicidad de elementos positivos que aporta en la vida de las personas (London y Formichella, 2006).

Entonces, la falta de educación puede implicar importantes privaciones (Sen, 2003). Por ello, que el origen socioeconómico de los jóvenes sea una influencia clave en sus trayectorias escolares sugiere que la educación puede constituir un mecanismo reproductor de injusticia. Dado que no todos los hogares disponen de los recursos económicos y humanos necesarios para que sus hijos se eduquen, algunos podrían caer en una trampa o mecanismo auto-reforzado que haga que la pobreza persista (Barham y otros, 1995; Berti Ceroni, 2001, Santos, 2007a; Formichella, 2009).

Ahora bien, como enfatizan Longás Mayayo y otros (2016), más allá de la asociación ampliamente demostrada entre el nivel socioeconómico (NSE) y el éxito escolar, existen otros factores que influyen en este complejo fenómeno. Resulta de interés, entonces, identificar otros elementos que puedan favorecer el desarrollo de trayectorias deseables aún en entornos familiares vulnerables. Esto constituiría un aporte para pensar en estrategias, complementarias a la ineludible acción en la esfera socioeconómica, que favorezcan la igualdad de oportunidades educativas.

Estas consideraciones han motivado el estudio de las oportunidades educativas de los niños y adolescentes que habitan en contextos de 
vulnerabilidad y la intención de mejorarlas, a través del Proyecto de Desarrollo Tecnológico y Social (PDTS): "Diseño de estrategias para mejorar las oportunidades educativas de la población vulnerable de Bahía Blanca a través de la ONG Red de Voluntarios"2.

A modo de contextualización, cabe mencionar que la ciudad de Bahía Blanca es la cabecera del partido homónimo y se encuentra ubicada al sudoeste de la provincia de Buenos Aires, Argentina. Según el último Censo Nacional de Población y Viviendas, sus aproximadamente 300 mil habitantes la convierten en la cuarta localidad más poblada de la provincia. La ciudad cuenta con una ubicación clave por su condición portuaria y su funcionamiento como nodo energético-logístico, tiene un importante polo tecnológico y parque industrial, así como un sector de comercio y servicios desarrollado. El ingreso per cápita familiar promedio supera a la media del país, y la tasa de pobreza (según Necesidades Básicas Insatisfechas) es algo menor a la nacional. Asimismo, suele presentar menores tasas de subempleo, sobreocupación, informalidad e inestabilidad laboral que el resto del país ${ }^{3}$. Sin embargo, las tasas de desempleo suelen ser superiores al promedio nacional ${ }^{4} \mathrm{y}$ distintos indicadores socioeconómicos dan cuenta de persistentes dificultades experimentadas por la población.

Actualmente, una de las problemáticas sociales más relevantes es la marcada segregación urbana, tal que es posible identificar zonas según el uso del espacio, el valor de la tierra y el tipo de edificación predominante y los niveles de calidad de vida (CREEBBA, 2015; Prieto, 2007). Entre las zonas residenciales, los sectores socioeconómicos se agrupan constituyendo barrios de características más bien homogéneas. Así, como resume Malisani (2017), mientras que las zonas Centro y Norte albergan a la población de mayores recursos, en el Sur y Oeste de la ciudad se concentran los segmentos más vulnerables. Estos barrios carecen de infraestructura y servicios básicos de vivienda, y suelen padecer problemas de accesibilidad y ambientales. Asimismo, predominan las viviendas de mala calidad constructiva y con hacinamiento.

\footnotetext{
2 El proyecto está dirigido por la Dra. Silvia London e integra a docentes e investigadores del IIESS (CONICET), del Departamento de Economía (UNS), de la Universidad Tecnológica Nacional y de la Universidad Provincial del Sudoeste.

${ }^{3}$ Estimaciones propias empleando la Encuesta Permanente de Hogares, IV trimestre del 2014.

${ }^{4}$ Ver, por ejemplo, el Boletín de Estadísticas Laborales de Bahía Blanca-Cerri de marzo del 2017 (Gustavo Burachik, Departamento de Economía, UNS).
} 
La ONG "Red de Voluntarios para una Infancia Mejor" desarrolla tareas de intervención con el fin de promocionar los derechos de la infancia y la adolescencia en barrios periféricos de la ciudad. En particular, pone énfasis en el derecho a la educación. En este contexto, el PDTS tiene como objetivo general evaluar de forma integral las condiciones socioeducativas en tres de los barrios en los que actúa la ONG, para luego diseñar estrategias orientadas a potenciar las actividades que ésta lleva a cabo como agente de desarrollo social.

Al igual que en el resto del país, puede afirmarse que en la ciudad la mayor parte de los gastos en la educación de los menores es solventada por sus propias familias y éstas -al disponer de desiguales recursos económicos, tiempo, acceso al crédito y posibilidades para hacer frente a los costos de oportunidad- tienen disímil capacidad de inversión (Barham y otros, 1995; Formichella, 2009; Krüger, Ibáñez Martín y Formichella, 2016). Asimismo, las familias difieren en el stock de capital humano que traen consigo (Llach, 2006; Santos, 2007b).

Lo anterior se relaciona con la noción de educabilidad, la cual hace referencia a aquellas condiciones sociales necesarias para que alguien asista a la escuela con éxito (López, 2006). Éstas se vinculan tanto con la manera en que la persona ha sido estimulada emocionalmente como con las características alimenticias y sanitarias de su hogar (Tedesco, 2000). Así, no todos los niños y jóvenes disponen del conjunto de elementos adecuados para articular con lo que les brinda la escuela y las características socioeconómicas de los alumnos constituyen la mayor influencia sobre los resultados educativos. La pertenencia a distintos estratos determina una adquisición diferenciada de capital económico y sociocultural, que se vincula con las posibilidades de acceder, permanecer y egresar del sistema educativo (Golovanevsky, 2006; Krüger, 2016; Riquelme y Herger, 2001).

Ahora bien, no todos los componentes del NSE familiar parecen tener la misma influencia en los resultados (Formichella 2011; Formichella y Krüger, 2013, 2016; Herrero, Peláez y González, 2007; Krüger, 2013; Longás Mayayo y otros, 2016; Marchionni, Pinto y Vázquez, 2013). En general, las estimaciones de la función de producción educativa muestran que el clima educativo del hogar y el estatus ocupacional tienen un efecto más significativo que el de las posesiones de riqueza, aunque sí suele aparecer como relevante la disponibilidad de recursos educativos, libros o posesiones culturales. 
Al mismo tiempo, como se analiza en Krüger y Formichella (2016), existen distintos atributos personales como las actitudes, expectativas y motivaciones -competencias no-cognitivas- que influyen en el rendimiento educativo. Estos factores pueden tener un rol como mediadores o moderadores del impacto del NSE familiar ya que, si bien son condicionados por el mismo, también se ven determinadas por otros factores contextuales relacionados con la institución escolar, los compañeros o el vecindario. Asimismo, las valoraciones, actitudes y expectativas de los padres, pueden representar un fuerte condicionamiento. Debe contemplarse, como analizan Bonal y Zancajo (2018), que si bien el contexto de pobreza provee un cierto habitus -que lleva a actuar y pensar de forma particular- no implica que todos aquellos que comparten ciertas condiciones de vida se comporten de la misma forma.

Afirman Longás Mayayo y otros (2016) que el éxito o fracaso escolar recibe la influencia de múltiples factores de diferentes niveles: desde el macrosistema (normas y políticas sociales), el exosistema (entornos a los que pertenecen las familias, como las comunidades) y el microsistema (familias, escuelas, compañeros). Es posible encontrar estudiantes resilientes en entornos vulnerables, que por sus cualidades no-cognitivas y por el tipo de apoyo que reciben desde la familia y otros ámbitos de socialización, como la escuela y la comunidad, logran alcanzar resultados inesperadamente positivos para su contexto de pertenencia.

En nuestro país, existen estudios que analizan la situación educativa de niños y jóvenes en poblaciones vulnerables. Para la Ciudad de Buenos Aires, Krichesky y otros (2011) indagan, desde una perspectiva cualitativa, en las percepciones y experiencias educativas de jóvenes de origen social carenciado en distintos contextos institucionales. Encuentran, por un lado, que el abandono y la repitencia son problemas centrales en sus trayectorias educativas y se asocian con la precariedad en las condiciones de vida; por el otro, que la escuela es un espacio significativo en la vida de los jóvenes, y que los saberes más apreciados -que a su vez posibilitarían la supervivencia en la escuela- se vinculan con la adquisición de valores, la responsabilidad, o la preparación para el futuro. Feijoó y Corbetta (2004) analizan los desafíos educativos en escenarios de pobreza del conurbano bonaerense y enfatizan la necesidad de fortalecer la educabilidad de los chicos, atendiendo a las condiciones materiales de vida, promoviendo la relación escuela-familia, integrando a la educación en la vida barrial y construyendo confianza básica y expectativas de logro. Guevara (2016) considera la escolarización secundaria en contextos de pobreza 
en las afueras de La Plata desde la perspectiva de los actores, principalmente las familias. Da cuenta de distintas estrategias que aplican los padres para reforzar las trayectorias escolares de sus hijos, de sus expectativas y valoraciones, y concluye que es clave considerar a las familias en la investigación sobre la escuela secundaria.

Sin dudas la agenda de investigación en torno a estas temáticas en el país es aún muy amplia, especialmente en el contexto de la ciudad de Bahía Blanca, y el presente trabajo pretende constituir un aporte a la comprensión del problema.

Los objetivos específicos del mismo son: i)conocer si la población de tres barrios periféricos, si bien es vulnerable en relación al resto de la ciudad, está conformada por grupos diferenciados en sus condiciones socioeconómicas; ii) comparar indicadores de inclusión educativa entre dichos grupos y al interior de los mismos -distinguiendo entre quienes tienen edad de asistir a los distintos niveles educativos-; iii) analizar si algunos niños o adolescentes alcanzan resultados inesperados (positivos o negativos) en relación al grupo al cual pertenecen, e identificar factores personales, familiares y contextuales que podrían estar moderando el impacto del nivel socioeconómico. Se espera que los resultados obtenidos representen un insumo útil para el diseño de estrategias de intervención por parte de la ONG y los hacedores de políticas locales.

\section{Metodología}

\section{Diseño}

Se adopta una perspectiva metodológica cuantitativa, empleando información primaria recabada a través de una encuesta realizada para el proyecto PDTS, a una muestra de hogares estadísticamente representativa de los barrios seleccionados. El diseño es no experimental, de tipo correlacional descriptivo y transversal.

\section{Participantes}

El relevamiento fue llevado a cabo en abril del 2016 en tres barrios periféricos de la ciudad de Bahía Blanca: Stella Maris, Nueve de Noviembre y Cabré Moré. Dichos barrios se ubican muy próximos entre sí, a unos 
cinco kilómetros del micro-centro de la ciudad. Stella Maris es el de mayor antigüedad, mientras que los asentamientos lindantes Nueve de Noviembre y Cabré Moré son de más reciente conformación. La información secundaria disponible, el Censo 2010, solo permite aproximarnos a una caracterización de esta área, ya que la zona se ha modificado sustancialmente durante los últimos años. Igualmente, posibilita la realización de ciertas comparaciones que probablemente continúan vigente. En suma, estos barrios presentaban, en relación al resto de la ciudad: una mayor proporción de la población conformada por niños y adolescentes; una mayor participación de extranjeros; un nivel educativo de los habitantes inferior; una tasa de desocupación más elevada; un porcentaje de hogares con NBI que triplicaba al valor general; una calidad constructiva y acceso a servicios básicos de electricidad, agua y gas inferiores; mayores condiciones de vulnerabilidad socioeconómica para los niños, y mayor inasistencia a tramos de escolaridad obligatoria.

Las encuestas fueron respondidas por miembros adultos de los hogares seleccionados. Cabe señalar que, previamente a que los encuestadores comiencen las visitas a dichos hogares, el equipo del proyecto hizo contacto con los referentes de los barrios para dar difusión al relevamiento. De este modo, cuando los hogares eran visitados no eran tomados por sorpresa y conocían los objetivos de la encuesta. Además, la decisión de responder o no estaba en cada individuo encuestado, a quien se le consultaba si deseaba participar antes de comenzar a hacerle las preguntas.

\section{Instrumentos}

El instrumento empleado consta de un cuestionario de hogar y otro de individuos. El primero, recaba información sobre el hogar y la vivienda, así como datos básicos sobre los miembros que lo componen. El segundo, recaba información sobre los individuos de 3 a 18 años (situación educativa actual, trayectoria educativa, disposición de recursos y condiciones para la escuela y el estudio, percepciones y expectativas con respecto a la escuela y su educación, etc.).

Dichos cuestionarios fueron desarrollados por el equipo de investigación del PDTS, adaptando distintos cuestionarios de relevamientos nacionales e internacionales a los objetivos del proyecto. Se consideraron, por ejemplo, los cuestionarios del Programa para la Evaluación Internacional de Alumnos (PISA) de la OCDE, del Tercer Estudio Regional Comparativo y Explicativo (TERCE) del LLECE-UNESCO, de la Encuesta 
Permanente de Hogares (EPH) y la Encuesta Nacional sobre Acceso y Uso de Tecnologías de la Información y Comunicación (ENTIC) del INDEC, Argentina.

La información recabada a partir de este instrumento permitió construir las siguientes variables, que se clasifican en tres grupos según su función en el análisis desarrollado:

Nivel socioeconómico de los hogares:

- Clima educativo del hogar: promedio de años de escolarización de los miembros de 18 años y más.

- Máximo nivel educativo alcanzado por los miembros del hogar

- Ingresos totales mensuales del hogar

- Pobreza e indigencia por ingresos: cálculos realizados a partir de la Canasta Básica de Alimentos y la Canasta Básica Total publicadas por el INDEC para abril del 2016.

- Necesidades básicas insatisfechas: vivienda de tipo inconveniente (NBI1); falta de agua potable en el interior de la vivienda (NBIlbis); ausencia de retrete o retrete sin arrastre de agua (NBI2); hacinamiento o más de tres personas por ambiente (NBI3); presencia de niños de 6 a 12 años que no asisten a la escuela (NBI4); presencia de niños y jóvenes en edad de escolaridad obligatoria que no asisten a la escuela (NBI4bis); hogar sin miembros ocupados o jubilados, o bien con más de tres miembros por persona ocupada o jubilada, cuyo jefe tiene como máximo nivel educativo la primaria incompleta (NBI5). Finalmente, si el hogar presenta al menos una de estas NBI, la variable NBIbis toma valor 1 .

- Características del jefe del hogar: estabilidad laboral, desocupación, inactividad, nivel educativo.

- Posesión de bienes y acceso a servicios: auto, conexión a internet y TV por cable.

- Consumo de alimentos de mayor precio y valor nutritivo: carnes, verduras y lácteos.

Resultados o situación educativa de los niños y jóvenes:

- Asistencia en el momento actual

- Abandono del colegio y sus motivos 
- Repitencia y cantidad de años repetidos

- Cantidad de veces en que el niño o joven llegó tarde y cantidad de veces en que faltó a la institución educativa durante las dos semanas previas a la encuesta.

- Motivos principales para el ausentismo o la impuntualidad

Posibles variables mediadoras entre el NSE y los resultados educativos:

Características del hogar, de la institución, la trayectoria educativa y de los niños y jóvenes que podrían actuar como moderadoras de la influencia del NSE y podrían explicar situaciones de resiliencia en contextos adversos. Se analizan en el trabajo las siguientes:

Variables vinculadas a los hogares:

- Hogar con cónyuge

- Hogar con jefa mujer

- Condiciones del hogar vinculadas al estudio (disposición de un lugar para estudiar, de luz para leer y de un lugar para guardar elementos de estudio).

- Frecuencia con que en el hogar realizan ciertas actividades (leer, jugar videojuegos, ir al cine o teatro, ver televisión, comentar las noticias, hacer deportes, navegar en Internet).

- Disposición de materiales vinculados al estudio (libros, manuales, diccionario).

- Si el niño o joven trabaja más de dos horas diarias en tareas domésticas.

- Si los padres acompañan en las tareas escolares.

- Si el estudiante va solo o acompañado a la institución escolar.

Variables vinculadas a la institución escolar y la trayectoria educativa:

- Si el estudiante recibe comida diaria en la institución.

- Si el estudiante asiste a jornada extendida o completa.

- Si el estudiante cambió de escuela (pero no por conclusión de ciclo escolar).

- Si el estudiante fue a maternal.

- Si el estudiante fue a nivel inicial.

- Si el estudiante usa libros en la escuela.

- Si el estudiante usa PC en la escuela. 
- Distancia a la escuela.

- Turno de asistencia a la escuela.

Variables vinculadas a los niños y jóvenes:

- Si usa computadora y si lo hace para tareas educativas.

- Si va a clases particulares.

- Horas promedio de estudio semanales.

\section{Procedimiento}

Se realizó un diseño muestral bietápico y probabilístico: la unidad primaria de muestreo se conformó por los polígonos de las manzanas de cada barrio y los hogares constituyeron la unidad secundaria de muestreo. Los totales poblacionales se estimaron aplicando factores de re-ponderación considerando la falta de respuesta. Se relevaron finalmente 381 hogares y 1459 personas que, luego de aplicar el ponderador muestral correspondiente, representan un total de 1077 hogares y 4125 personas.

\section{Análisis de datos}

La estrategia de análisis escogida consta de tres pasos principales:

a) Para detectar si la población de los barrios de interés está conformada por grupos diferenciados en cuanto a sus condiciones socioeconómicas, se aplicó un Análisis de Conglomerados a partir de una selección de indicadores de NSE de los hogares. Luego, los hogares de cada conglomerado fueron caracterizados a partir del conjunto completo de variables de NSE.

b) Se comparó la situación educativa de los niños y jóvenes pertenecientes a cada conglomerado, distinguiendo entre aquellos que tienen edad de asistir al nivel inicial, primario o secundario.

c) Finalmente, se identificaron aquellos casos en los que los niños y jóvenes presentaban resultados educativos inferiores o superiores a los de su conglomerado, y se buscaron posibles variables moderadoras que permitieran explicar esta cuestión. 
El análisis de conglomerados es una técnica estadística no paramétrica para reorganizar un conjunto de elementos en grupos que tienen un alto grado de "asociación natural" (Aldenderfer y Blashfield, 1984). Es un procedimiento que permite realizar análisis exploratorios, aportando clasificaciones de los datos para extraer conclusiones relevantes o bien servir como paso previo para análisis inferenciales posteriores. En este caso, se buscó agrupar a los hogares relevados según su proximidad respecto de tres indicadores de su NSE: el clima educativo, el ingreso mensual total y la cantidad de NBI presentes (exceptuando NBI4 y NBI4bis). Previamente, las variables fueron estandarizadas para quitar la influencia de su dispersión y unidad de medida, y se removieron los valores atípicos u observaciones con un desvío estándar mayor a 3 (en valor absoluto). En el caso de que las observaciones tuvieran valores perdidos en alguna de las variables de agrupación, su asignación se realizó a partir de los valores de las variables sin datos faltantes.

Se aplicó un algoritmo de aglomeración no jerárquico, el popular método de k-medias de MacQueen, escogido por su simplicidad y eficiencia. Este algoritmo realiza una partición inicial de k-elementos que forman los centroides o medias iniciales, y luego va asignando al resto de las observaciones al grupo con el centroide más cercano. La cercanía entre cada par de observaciones se definió a partir de la distancia Euclídia, que agrega las diferencias, elevadas al cuadrado, entre cada una de las variables. El objetivo final es minimizar la varianza intra-grupo y maximizar la varianza entre grupos (Anderberg, 1973).

El número óptimo de conglomerados se definió maximizando el índice de Calinski y Harabasz (1974), o pseudo-F, criterio recomendado por Milligan y Cooper (1985) y Savovay otros (2006). Este índice se define de la siguiente manera:

$$
C H_{k}=\frac{S S_{B} / k-1}{S S_{W} / n-k}
$$

Donde $k$ es el número de conglomerados, $n$ es el número de observaciones, $S S_{B}$ es la varianza total entre grupos y $S S_{W}$ es la varianza total dentro de cada grupo.

Una vez agrupados los hogares en conglomerados, los siguientes pasos del análisis se realizaron a través de estadísticas descriptivas, evaluándose la asociación entre variables a partir de pruebas de diferencias de medias o contrastes de Chi-cuadrado. 


\section{Resultados}

\section{Análisis de conglomerados}

La maximización del índice de Calinski y Harabasz (1974) indicó una partición óptima de dos conglomerados de hogares ${ }^{5}$. El estadístico F reportado en la tabla ANOVA permitió comprobar que las tres variables seleccionadas resultaron estadísticamente significativas en la configuración de los conglomerados, siendo la más influyente el clima educativo de los hogares ${ }^{6}$.

El primer conglomerado se compone de 161 hogares y el segundo de 206. Al primero pertenecen 575 individuos que, ponderados, representan una población de 1659 personas. El segundo conglomerado se compone de 825 individuos, que representan una población total de 2317.

Si bien toda la muestra proviene de un sector de la ciudad que presenta carencias en diversos aspectos socioeconómicos, el análisis realizado permite identificar dos grupos de hogares cuyos indicadores de NSE difieren significativamente. El primer conglomerado agrupa hogares que, en promedio, presentan condiciones relativamente más precarias que las del segundo conglomerado. Por ello, a continuación los denominaremos "Conglomerado menos favorable" y "Conglomerado más favorable", respectivamente.

La información presentada en la Tabla 1 permite caracterizar ambos conglomerados a partir de sus valores promedio para distintas variables indicadoras del NSE de los hogares.

Los datos del relevamiento confirman que los barrios considerados poseen un bajo NSE. A su vez, puede observarse que los hogares pertenecientes al conglomerado de menor NSE se encuentran en mayor desventaja en relación con el nivel educativo de sus miembros adultos, a los ingresos mensuales y a la presencia de NBI. En este caso, un $27 \%$ de los hogares tiene un clima educativo bajo (menor a 6 años) y sólo un $6 \%$ cuenta con un clima educativo medio-alto (de 10 a 12 años). En cambio, en el conglomerado de mayor NSE, sólo un 1\% tiene un clima educativo

\footnotetext{
${ }^{5}$ Los valores respectivos del índice fueron: 142,50 para $\mathrm{k}=2 ; 125,8$ para $\mathrm{k}=3 ; 140,61$ para $\mathrm{k}=4$; $\mathrm{y} 141,91$ para $\mathrm{k}=5$.

${ }^{6}$ Los estadísticos F fueron los siguientes: cantidad de NBI $(33,9)$; clima educativo $(453,6)$; ingreso mensual total $(65,7)$. Los p-values resultaron menores a 0,001 en todos los casos.
} 
bajo, un $58 \%$ tiene un clima educativo medio-alto y un $17,5 \%$ llega a tener un clima educativo alto (12 años o más).

Los ingresos mensuales de los hogares son en general más bajos que su línea de pobreza (72\%), y el nivel total de indigencia es alarmante (20\%). A su vez, el primer grupo se encuentra en una posición más precaria: con un $86 \%$ de hogares pobres y un $34 \%$ de hogares indigentes, frente a niveles respectivos del $65 \%$ y el $11 \%$ en el segundo grupo.

Con respecto a las NBI, la vivienda de tipo inconveniente (NBI1), las deficientes condiciones sanitarias (NBI2) y el hacinamiento (NBI3) son las más acuciantes en general, y resultan claramente más relevantes en el primer conglomerado. En total, un $60 \%$ de estos hogares presenta al menos una NBI.

Otras variables disponibles a partir del relevamiento permiten completar el cuadro de situación. Los jefes de hogar del grupo desfavorable se encuentran en una peor posición en cuanto a su nivel de estudios, la estabilidad de su trabajo o la inactividad. Asimismo, sólo algunos de estos hogares poseen cobertura médica propia y acceso a ciertos bienes o servicios como autos, conexión a internet y TV por cable. Finalmente, algunas preguntas del cuestionario permiten captar aproximadamente la calidad de la alimentación de las familias encuestadas, la cual puede incidir en el desarrollo y la salud de los niños. Los datos confirman lo esperado: en el conglomerado menos favorable los hogares reportan un menor consumo de lácteos, carnes y verduras, alimentos que tienen un mayor valor nutritivo, pero resultan menos asequibles. 
Tabla 1 Caracterización de los conglomerados según el NSE de los hogares

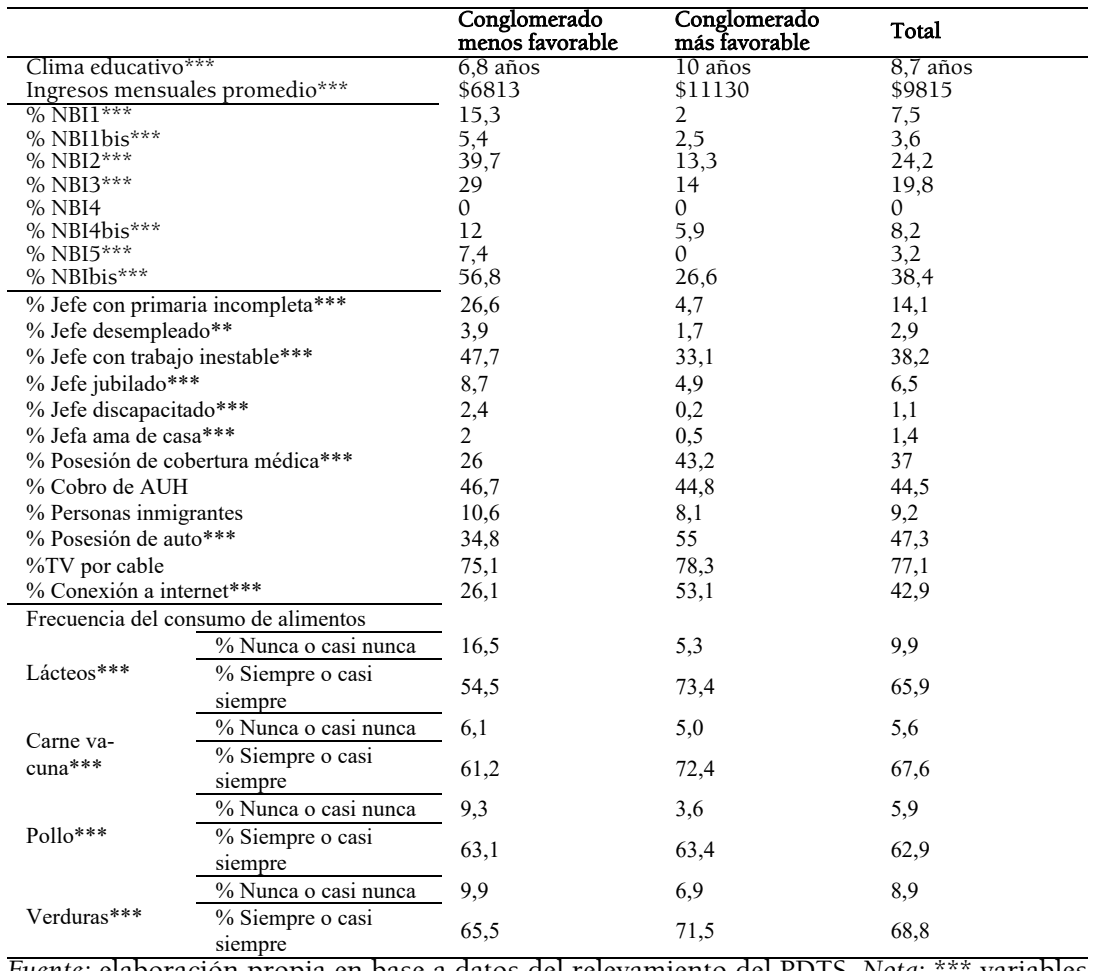

Fuente: elaboración propia en base a datos del relevamiento del PDTS. Nota: *** variables cuantitativas: la diferencia de medias entre ambos conglomerados es significativa al $1 \%$; variables cualitativas: la asociación con el conglomerado de pertenencia es significativa al $1 \%$ según la prueba Chi-2; ** significatividad al $5 \%$.

\section{Comparación de la situación educativa entre ambos conglomerados}

El segundo paso de la estrategia de análisis es la comparación de la situación educativa de los niños y jóvenes pertenecientes a cada conglomerado de hogares, para lo cual se los agrupó previamente según su edad. Entre los niños de 3 a 5 años, en edad de asistir al nivel inicial, 126 pertenecen al conglomerado menos favorable y 169 al más favorable. Entre los niños en edad de asistir al nivel primario, de 6 a 11 años, 210 pertenecen al primer grupo y 297 al segundo. Por último, entre los jóvenes de 12 a 18 años, en edad de asistir al secundario, 247 pertenecen al primer conglomerado y 313 al otro. 
Para quienes están en edad de asistir al nivel inicial, el aspecto más relevante es el acceso o la asistencia. En este caso, un 85\% de los niños de estos barrios se encuentra asistiendo a una institución educativa. Entre conglomerados, sin embargo, hay una diferencia apreciable y estadísticamente significativa: la tasa de asistencia para quienes provienen de hogares del cluster de menor NSE es del 77\%, mientras que para el cluster de mayor NSE es de $91 \%$.

Respecto de la impuntualidad o las faltas al jardín, no se observan diferencias significativas entre ambos conglomerados. Cabe destacar, igualmente, que a nivel general cerca de un $50 \%$ de los chicos faltaron tres o más veces durante las dos semanas previas a la encuesta, y esto se explicaría principalmente por problemas de salud, problemas de acceso a la institución (calles anegadas, dificultades con el transporte, etc.) y la no disponibilidad de un adulto para acompañarlos.

Entre aquellos que tienen edad de asistir al nivel primario, nadie reportó inasistencia, por lo que el resultado más interesante es el de la repitencia. Como se observa en la Tabla 2, un 22\% de quienes pertenecen al conglomerado menos favorable han repetido al menos una vez, mientras que sólo un $5 \%$ de los que pertenecen al conglomerado más favorable han atravesado esta dificultad. En ambos casos, en su mayoría la repitencia ha sido por única vez.

Tabla 2. Situación educativa por conglomerado - grupo etario de nivel primario

\begin{tabular}{lllll}
\hline & & $\begin{array}{l}\text { Conglomerado me- } \\
\text { nos favorable }\end{array}$ & $\begin{array}{l}\text { Conglomerado más } \\
\text { favorable }\end{array}$ & Total \\
\hline \% Asistencia & 100 & 100 & 100 \\
\% Repitencia*** & & 21,9 & 5,3 & 12,4 \\
\cline { 1 - 1 } Llegó tarde* $^{*}$ & $\begin{array}{l}\text { \% Dos veces o me- } \\
\text { nos }\end{array}$ & 94,4 & 96,85 & 96,2 \\
\cline { 1 - 2 } Faltón veces o más & 5,6 & 3,2 & 3,8 \\
& $\begin{array}{l}\text { \% Dos veces o me- } \\
\text { nos }\end{array}$ & 31,5 & 47,9 & 41,6 \\
& \% Tres veces o más & 68,5 & 52,1 & 58,4 \\
\hline
\end{tabular}

Fuente: elaboración propia en base a datos del relevamiento del PDTS. Nota: ${ }^{* * *}$ variables cuantitativas: la diferencia de medias entre ambos conglomerados es significativa al 1\%; variables cualitativas: la asociación con el conglomerado de pertenencia es significativa al 1\% según la prueba Chi-2; ** significatividad al 5\%.

Se registran diferencias entre los grupos en cuanto a la posibilidad de asistir a la escuela en tiempo y forma. Entre quienes pertenecen al conglomerado de menor NSE, un mayor porcentaje reporta haber llegado tarde tres o más veces durante las últimas dos semanas o haber faltado tres o más veces. Las causas no difieren significativamente entre ambos conglomerados, siendo la salud, los problemas de acceso y la falta de un 
adulto que los acompañe los principales motivos. Aparece un pequeño porcentaje, también, de reportes de inasistencia por falta de interés y por haber tenido que cuidar de un familiar.

Por su parte, los jóvenes en edad de asistir al nivel secundario reportan problemas de acceso, especialmente quienes provienen de hogares en el cluster menos favorable. Casi un $15 \%$ de los mismos indica que abandonó el colegio, frente a un $5 \%$ de quienes pertenecen al relativamente más favorable. Los motivos principalmente aducidos para el abandono son: falta de interés por el estudio (41\%); dificultad para el estudio (23\%); necesidad de trabajar fuera del hogar o realizar tareas de cuidado en el hogar (19\%) (ver Tabla 3).

Tabla 3. Situación educativa por conglomerado - grupo etario de nivel secundario

\begin{tabular}{|c|c|c|c|c|}
\hline & & $\begin{array}{l}\text { Conglomerado } \\
\text { menos favorable }\end{array}$ & $\begin{array}{l}\text { Conglomerado más } \\
\text { favorable }\end{array}$ & Total \\
\hline$\%$ Asistencia*** & & 85,4 & 94,2 & 90,4 \\
\hline$\%$ Abandono** & & 14,6 & 4,8 & \\
\hline$\%$ Repitencia** & & 58,1 & 37,9 & 46,5 \\
\hline \multirow{2}{*}{ Llegó tarde* } & $\begin{array}{l}\text { \% Dos veces o me- } \\
\text { nos }\end{array}$ & 89,3 & 96,9 & 93,95 \\
\hline & \% Tres veces o más & 10,7 & 3,15 & 6,1 \\
\hline \multirow{2}{*}{ Faltó } & $\begin{array}{l}\text { \% Dos veces o me- } \\
\text { nos }\end{array}$ & 60 & 54,1 & 55,3 \\
\hline & $\%$ Tres veces o más & 40 & 45,9 & 44,7 \\
\hline
\end{tabular}

Fuente: elaboración propia en base a datos del relevamiento del PDTS. Nota: ${ }^{* * *}$ variables cuantitativas: la diferencia de medias entre ambos conglomerados es significativa al 1\%; variables cualitativas: la asociación con el conglomerado de pertenencia es significativa al 1\% según la prueba Chi-2; ** significatividad al 5\%.

Respecto de la repitencia, también hay diferencias significativas entre los grupos, siendo el de menor NSE más afectado por este problema. Respecto de la cantidad de veces que han repetido aquellos que lo han hecho, los clusters no difieren significativamente, pero en total resulta preocupante que un $40 \%$ de los jóvenes ha repetido al menos 2 años.

En el mismo sentido, los jóvenes del conglomerado más pobre reportan haber llegado más veces tarde al colegio, mientras que las diferencias en la cantidad de faltas no son significativas estadísticamente. Los motivos principales reportados son nuevamente la salud y los problemas de acceso, pero aparecen con mayor peso la necesidad de quedarse en casa para cuidar de alguien y la falta de interés por la escuela.

Entonces, la comparación de ambos conglomerados indica que el NSE resulta un determinante crucial de la situación educativa, ya que aún en un entorno desfavorecido las diferencias relativas tienen una incidencia significativa. Ahora bien, se visualiza cierta variabilidad al interior de 
cada conglomerado: el tipo de problemas o formas de exclusión van variando según el nivel educativo y, a su vez, hay niños y jóvenes que presentan resultados mejores o peores que los predominantes en su grupo, cuestión que se explora en la siguiente sección.

\section{Análisis de grupos específicos}

Resulta particularmente interesante analizar dos grupos de niños y jóvenes: por un lado, aquellos que perteneciendo al conglomerado menos favorecido en relación a las características socioeconómicas logran situarse en la cima de la distribución de resultados educativos y, por otra parte, aquellos que si bien pertenecen al conglomerado más favorecido obtienen el resultado más bajo.

Para poder observar a estos grupos en particular, primero es necesario identificarlos. Para ello, se crea una variable ordinal denominada Resultado, la cual toma cuatro valores posibles: (1) si el niño o joven no asiste a la escuela; (2) si asiste y ha repetido más de una vez; (3) si asiste y ha repetido sólo una vez; y (4) si asiste y nunca ha repetido. Se analiza la relación de esta variable Resultado con la variable que indica el conglomerado de pertenencia para cada observación (se consideran a los sujetos entre 6 y 18 años) y se encuentra que se rechaza al 1\% la Hipótesis Nula de independencia entre las observaciones según la prueba de Chi-2 de Pearson. Puede afirmarse entonces que existe un vínculo entre el cluster de pertenencia y el resultado educativo. Se arriba a la misma conclusión si se segmenta la muestra según franjas etarias ( 6 a 11 y 12 a 18).

Dado que la escolarización en los chicos que tienen edad de asistir al nivel primario es completa, la variable Resultado sólo admite valores de 2 a 4. Esto, sumado a que la variabilidad en los resultados restantes es baja en comparación con el grupo etario que se corresponde con el nivel medio (ver Tabla 4), hace que sea más interesante enfocarse en la franja etaria de 12 a 18 años.

De este modo, se pondrá atención en el 38,2\% de adolescentes que, si bien pertenecen al grupo relativamente menos favorable, asisten a la escuela y nunca han repetido, así como en el $5 \%$ de los adolescentes que, a pesar de pertenecer al grupo más favorable, no asisten. Para hacer más sencilla la lectura, se denomina (A) al primer caso y (B) al segundo. El objetivo de esta sección es, a partir de los grupos seleccionados, detectar qué variables son relevantes más allá del NSE y que podrían estar actuando como mediadoras entre éste y los resultados educativos. 
Tabla 4. Resultado educativo según conglomerado, por grupos de edad

\begin{tabular}{|c|c|c|c|c|c|c|}
\hline \multirow[b]{2}{*}{ Resultado } & \multicolumn{3}{|c|}{ Grupo etario de nivel primario } & \multicolumn{3}{|c|}{ Grupo etario de nivel secundario } \\
\hline & $\begin{array}{l}\text { Conglome- } \\
\text { rado menos } \\
\text { favorable }\end{array}$ & $\begin{array}{l}\text { Conglome- } \\
\text { rado más fa- } \\
\text { vorable }\end{array}$ & Total & $\begin{array}{l}\text { Conglome- } \\
\text { rado menos } \\
\text { favorable }\end{array}$ & $\begin{array}{l}\text { Conglome- } \\
\text { rado más fa- } \\
\text { vorable }\end{array}$ & Total \\
\hline$\%$ No asiste & 0 & 0 & 0 & 14,3 & $5(\mathrm{~B})$ & 9,1 \\
\hline $\begin{array}{l}\text { \% Asiste y re- } \\
\text { pitió más de } \\
\text { una vez }\end{array}$ & 1,4 & 0,8 & 1 & 21,7 & $13,5(\mathrm{C})$ & 16,9 \\
\hline $\begin{array}{l}\% \text { Asiste y re- } \\
\text { pitió sólo una } \\
\text { vez }\end{array}$ & 19,6 & 4,7 & 10,7 & 25,8 & 21,4 & 23,9 \\
\hline $\begin{array}{l}\% \text { Asiste y } \\
\text { nunca repitió }\end{array}$ & 79 & 94,5 & 88,3 & $38,2(\mathrm{~A})$ & 60,2 & 50,2 \\
\hline Total & 100 & 100 & 100 & 100 & 100 & 100 \\
\hline
\end{tabular}

En la Tabla 5, entonces, se comparan los valores de algunas variables relacionadas con el hogar del que provienen los adolescentes pertenecientes a cada uno de estos grupos.

Tabla 5. Comparación de variables del hogar entre los grupos (A) y (B)

\begin{tabular}{|c|c|c|c|}
\hline Variables del hogar & $\begin{array}{l}\text { Asiste y nunca re- } \\
\text { pitió-Conglome- } \\
\text { rado menos favo- } \\
\text { rable } \\
\text { (A) }\end{array}$ & $\begin{array}{l}\text { No asiste- Conglome- } \\
\text { rado más favorable } \\
\text { (B) }\end{array}$ & $\begin{array}{l}\text { Total adolescentes } \\
\text { entre } 12 \text { y } 18 \text { años }\end{array}$ \\
\hline \% Hogar con cónyuge & 83,9 & 59,5 & 81,2 \\
\hline$\%$ Hogar con jefe mujer & 51,8 & 40,5 & 35,1 \\
\hline $\begin{array}{l}\text { \% Trabajan más de dos horas diarias } \\
\text { en tareas domésticas }\end{array}$ & 35 & 53,1 & 34,4 \\
\hline $\begin{array}{l}\% \text { En el hogar hay un lugar para es- } \\
\text { tudiar }\end{array}$ & 78 & 48 & 63,9 \\
\hline $\begin{array}{l}\% \text { En el hogar siempre o casi siem- } \\
\text { pre leen }\end{array}$ & 39 & 17 & 39 \\
\hline $\begin{array}{l}\% \text { En el hogar siempre o casi siem- } \\
\text { pre juegan videojuegos }\end{array}$ & 23,8 & 55,8 & 32,3 \\
\hline \% En el hogar siempre o casi siem- & 2,7 & 0 & 7,4 \\
\hline $\begin{array}{l}\text { \% En el hogar siempre o casi siem- } \\
\text { pre miran televisión }\end{array}$ & 82,2 & 100 & 85 \\
\hline $\begin{array}{l}\% \text { En el hogar siempre o casi siem- } \\
\text { pre hacen deportes }\end{array}$ & 64,1 & 17 & 45,4 \\
\hline $\begin{array}{l}\% \text { En el hogar siempre o casi siem- } \\
\text { pre navegan en Internet }\end{array}$ & 59,1 & 100 & 60,3 \\
\hline
\end{tabular}

Fuente: elaboración propia en base a datos del relevamiento del PDTS.

En principio, se detectan algunas cuestiones que caracterizan de forma diferenciada a los dos grupos considerados y que podrían dar indicios del porqué de la particularidad que comparten: presentar un resultado contrario al predominante en su cluster de pertenencia. Se destaca que en el grupo (A) es mayor, en término relativos y en comparación con el grupo (B), la proporción de adolescentes que habitan en hogares en los 
que están presentes ambos cónyuges o que la jefa es mujer. Incluso, dicha proporción es mayor que para el total de los jóvenes entre 12 y 18 años.

Por otra parte, pueden analizarse las diferencias en relación con el tipo de actividades que más se realizan en los hogares: en el grupo (A) es menor la proporción (en relación al grupo B), que juegan videojuegos, miran televisión o utilizan internet. Mientras que en el grupo (B) es menor la proporción que frecuentemente hace deportes, va al cine o lee.

Se destaca que en el grupo (A) es mayor la proporción de chicos que posee en su casa un lugar para estudiar. Es decir, a pesar de tener mayores dificultades socioeconómicas que el grupo (B), las familias destinan más frecuentemente un espacio para el estudio, lo cual podría ser un indicador de la importancia que le asignan a la educación. Asimismo, en el grupo (A) es menos habitual encontrar adolescentes que destinen más de dos horas diarias a tareas domésticas, sugiriendo que en la organización del hogar se tiene en cuenta que no destinen demasiado tiempo a dichas tareas y dispongan de más tiempo para desarrollar otro tipo de actividades, entre ellas estudiar.

También resulta interesante comparar valores de variables vinculadas a las escuelas a las que asisten los jóvenes, a sus trayectorias educativas o a las actitudes que ellos y sus familias tienen en relación con la educación y/o institución escolar. Sin embargo, en este caso sólo se dispone de información para aquellos que asistían al colegio al momento de llevarse a cabo el relevamiento, por lo que no puede utilizarse en el análisis el grupo denominado (B). Por tal motivo, se compara el grupo (A) con aquellos adolescentes que asisten a la escuela y, si bien pertenecen al conglomerado de mayor NSE, obtienen el peor de los resultados entre los que asisten (han repetido más de una vez). A este grupo se lo denomina (C).

En primer lugar, se visualiza que cierta proporción de quienes pertenecen al grupo (A) reciben asistencia alimenticia en la escuela y asisten a jornada extendida o completa, mientras que ningún adolescente del grupo (C) lo hace (ver Tabla 6). También se observa que los primeros han tenido mayor estabilidad escolar, tanto en relación grupo (C) como al total de los alumnos, ya que se encuentra con menor frecuencia que hayan cambiado de escuela. Asimismo, se destaca que es relativamente mayor la proporción del grupo (A) que utiliza computadora en la escuela. 
Tabla 6. Comparación de variables vinculadas a la institución educativa y a la trayectoria escolar entre los grupos (A) y (C)

\begin{tabular}{lll}
\hline Variables de la institución educativa y la trayectoria escolar & $\begin{array}{l}\text { Asiste y nunca repitió-Con- } \\
\text { glomerado menos favorable } \\
\text { (A) }\end{array}$ & $\begin{array}{l}\text { Asiste y repitió más de } \\
\text { una vez-Conglomerado } \\
\text { más favorable } \\
\text { (C) }\end{array}$ \\
\hline $\begin{array}{l}\text { \% que recibe comida diaria en la institución } \\
\text { \% que asiste a jornada extendida o completa }\end{array}$ & $\begin{array}{l}\text { Total adolescentes entre } \\
12 \text { y 18 años que asisten } \\
\text { al colegio }\end{array}$ & 0 \\
$\begin{array}{l}\text { escolar) } \\
\text { \% que fue a maternal }\end{array}$ & 12,5 & 0 \\
\% que fue a nivel inicial & 40,2 & 76,4 \\
\% que usa libros en la escuela & 11,9 & 8,7 \\
$\%$ que usa PC en la escuela & 96,8 & 100 \\
\hline
\end{tabular}

Fuente: elaboración propia en base a datos del relevamiento del PDTS.

Por otro lado, se observa que los que pertenecen al grupo (A) asisten, en mayor proporción que los que pertenecen al grupo (C), a escuelas lejanas (más de 20 cuadras) y en el turno de mañana (ver Tabla 7). Una hipótesis que valdrá la pena contrastar en futuros análisis es que la asistencia a escuelas más alejadas, la cual requiere un mayor esfuerzo en términos del tiempo y costo del transporte, podría estar reflejando una mayor preocupación por parte de las familias por enviar a sus hijos a escuelas percibidas como de mayor calidad.

Tabla 7. Comparación de la distancia a la escuela y el turno escolar entre los grupos (A) y (C)

\begin{tabular}{|c|c|c|c|c|}
\hline & & $\begin{array}{l}\text { Asiste y nunca repi- } \\
\text { tió-Conglomerado } \\
\text { menos favorable } \\
\text { (A) }\end{array}$ & $\begin{array}{l}\text { Asiste y repitió más de } \\
\text { una vez-Conglomerado } \\
\text { más favorable } \\
\text { (C) }\end{array}$ & $\begin{array}{l}\text { Total adolescentes } \\
\text { entre } 12 \text { y } 18 \text { años } \\
\text { que asisten al cole- } \\
\text { gio }\end{array}$ \\
\hline \multirow{5}{*}{$\begin{array}{l}\text { Cantidad de cua- } \\
\text { dras a la escuela }\end{array}$} & $\%$ menos de 3 cuadras & 0 & 0 & 0 \\
\hline & $\%$ entre 3 y 5 cuadras & 0 & 0 & 0,9 \\
\hline & $\%$ entre 6 y 10 cuadras & 31,6 & 30,2 & 21 \\
\hline & $\%$ entre 11 y 20 cuadras & 15,7 & 38,4 & 32,3 \\
\hline & \% más de 20 cuadras & 52,7 & 31,4 & 45,8 \\
\hline \multirow{4}{*}{ Turno escolar } & \% Mañana & 86,3 & 26,1 & 66,8 \\
\hline & $\%$ Tarde & 8,9 & 46,4 & 26,3 \\
\hline & $\%$ Vespertino & 4,8 & & 0.9 \\
\hline & $\%$ Noche & 0 & 27,5 & 5,9 \\
\hline
\end{tabular}

Fuente: elaboración propia en base a datos del relevamiento del PDTS.

Con respecto a los hogares en los que habitan los estudiantes de estos dos grupos (ver Tabla 8), se destaca nuevamente que es más frecuente hallar adolescentes que tienen un lugar para estudiar en el grupo (A), tanto en relación con el grupo $(\mathrm{C})$ como al total de los estudiantes. Respecto a la posesión de elementos para estudiar (luz, libros, manuales y diccionarios), no se observan grandes diferencias. Sí cabe destacar la discrepancia registrada en relación con el acompañamiento de los padres en 
la realización de las tareas: mientras que en el grupo (A) están presentes en un $73,5 \%$ de los casos, en el grupo (C) apenas llegan a la mitad. Incluso la presencia en el grupo (A) es mayor que la correspondiente al grupo total de los estudiantes. Esto sugiere que los padres tienen un rol importante que cumplir. En el mismo sentido, se observa que en el grupo (A) es mucho menos frecuente que en el (C) que los estudiantes vayan solos hasta la institución escolar. En síntesis, el acompañamiento de los adultos se detecta como un factor relevante vinculado al logro de buenos resultados educativos en los menores.

Tabla 8. Comparación de variables del hogar entre los grupos (A) y (C)

\begin{tabular}{|c|c|c|c|}
\hline Variables vinculadas al hogar & $\begin{array}{l}\text { Asiste y } \\
\text { nunca repitió- } \\
\text { Conglome- } \\
\text { rado menos } \\
\text { favorable } \\
\text { (A) }\end{array}$ & $\begin{array}{l}\text { Asiste y repi- } \\
\text { tió más de } \\
\text { una vez-Con- } \\
\text { glomerado } \\
\text { más favorable } \\
\text { (C) }\end{array}$ & $\begin{array}{l}\text { Total ado- } \\
\text { lescentes } \\
\text { entre } 12 \text { y } \\
18 \text { años } \\
\text { que asisten } \\
\text { al colegio }\end{array}$ \\
\hline \% Lugar para estudiar & 78 & 26,7 & 63,3 \\
\hline $\begin{array}{l}\text { \% Que dispone de un lugar para guardar elementos } \\
\text { para el estudio en su hogar }\end{array}$ & 75,8 & 81,8 & 73 \\
\hline$\%$ Buena luz para leer & 96,1 & 88,8 & 95,6 \\
\hline$\%$ Libros de poesía o literatura & 67,2 & 77,7 & 71,3 \\
\hline$\%$ Textos o manuales & 76,8 & 70,6 & 81,3 \\
\hline$\%$ Diccionario & 85,5 & 88,1 & 87,2 \\
\hline$\%$ Cuyos padres los ayudan con la tarea & 73,5 & 50,1 & 66,5 \\
\hline Trayecto hacia la institu- $\quad \%$ Solo & 30,5 & 55,6 & 33,1 \\
\hline \% Acompañado & 69,5 & 44,4 & 66,9 \\
\hline
\end{tabular}

Fuente: elaboración propia en base a datos del relevamiento del PDTS.

Por último, cabe estudiar algunas variables relacionadas a los estudiantes. Por un lado, en el grupo (A) resulta más frecuente que en el (C) que los adolescentes asistan a clases particulares. Asimismo, las horas promedio de estudio son mayores y, finalmente, en relación con el uso de computadora, no se observan discrepancias porcentuales relevantes (ver Tabla 9).

Tabla 9. Comparación de variables individuales entre los grupos (A) y (C)

\begin{tabular}{|c|c|c|c|}
\hline Variables individuales & $\begin{array}{l}\text { Asiste y nunca repitió-Con- } \\
\text { glomerado menos favorable } \\
\text { (A) }\end{array}$ & $\begin{array}{l}\text { Asiste y repitió más de } \\
\text { una vez-Conglomerado } \\
\text { más favorable } \\
\text { (C) }\end{array}$ & $\begin{array}{l}\text { Total adolescentes entre } 12 \\
\text { y } 18 \text { años que asisten al co- } \\
\text { legio }\end{array}$ \\
\hline$\%$ que va a clases particulares & 20,8 & 7,4 & 23,1 \\
\hline Horas promedio de estudio por semana & 7 & 5,5 & 7,7 \\
\hline$\%$ que utiliza computadora & 80,1 & 72,4 & 76,3 \\
\hline $\begin{array}{l}\% \text { que utiliza computadora } \\
\text { para actividades educativas }\end{array}$ & 58,1 & 59,4 & 58,2 \\
\hline
\end{tabular}

Fuente: elaboración propia en base a datos del relevamiento del PDTS. 


\section{Discusión}

A partir de los resultados, se ha detectado que las condiciones socioeconómicas heterogéneas de los hogares representan diferencias que son lo suficientemente relevantes como para determinar situaciones educativas dispares entre los niños y jóvenes que los habitan. El conglomerado menos favorable presenta, en promedio, mayores dificultades vinculadas con el acceso y la permanencia en la escuela. Esto coincide con lo planteado por Barham y otros (1995), Berti Ceroni (2001), Santos (2007a) y Formichella (2009); quienes señalan que puede llegar a evidenciarse una trampa de pobreza en educación.

Asimismo, se observa que las condiciones socioeconómicas parecen incidir de manera diferenciada según la edad de los niños. Mientras que la asistencia se ve más condicionada por el origen social en los niveles inicial y secundario, en el nivel primario la cobertura es prácticamente universal. Además, según lo reportado por los encuestados, la impuntualidad y el ausentismo se vinculan con el NSE relativo desde la primaria en adelante, al igual que la repitencia, resultado que se va agravando o acumulando durante la trayectoria escolar.

El factor edad se asocia estrechamente con la cuestión de las condiciones socioeconómicas. Entre los estudiantes que habitan hogares pobres, los más grandes comienzan a sentir la presión de realizar tareas domésticas en dichos hogares, y/o ingresar al mercado laboral y contribuir con la manutención de sus miembros. El tiempo es un recurso limitado y los adolescentes, en especial los de mayor edad, poseen un mayor costo de oportunidad vinculado a dedicar tiempo al estudio en desmedro del trabajo, ya sea remunerado o no remunerado en sus propias viviendas. En este último caso, el hecho de que colaboren en las tareas domésticas, en especial las de cuidado, libera a otros miembros para que puedan participar del mercado laboral. Por ello, Feijoo y Corbeta (2004) resaltan la importancia de atender a las condiciones de supervivencia material y de promover vínculos entre la escuela y las familias.

Ahora bien, más allá de la incidencia del NSE, se han hallado otros aspectos que influyen sobre los resultados educativos, formando también parte de las denominadas condiciones de educabilidad (Lopez, 2006). Es decir, tal como señalan Longás Mayayo y otros (2016), existen múltiples factores que influyen en el complejo proceso de enseñanza y aprendizaje. Aquí, se han detectado las siguientes cuestiones vinculadas a los hogares: 
los adolescentes que habitan en aquellos en los que están presentes ambos cónyuges, la jefa es mujer, poseen un lugar para estudiar, tienen menos responsabilidades en las tareas domésticas y/o reciben mayor acompañamiento de los padres en las tareas y/o trayecto hasta la escuela; logran mejores resultados que quiénes habitan hogares con condiciones inversas. En este mismo sentido, Guevara (2016) hace hincapié en la necesidad de tener en cuenta a las familias a la hora de analizar la problemática educativa de nivel medio.

Además, el hallazgo descrito en el párrafo anterior se condice con la perspectiva adoptada por Bonal y Zancajo (2018), según la cual la interpretación de los comportamientos humanos excede a las restricciones impuestas por la condición de pobreza. Es decir, las opciones se encuentran limitadas por la estructura de oportunidades disponibles, pero existe un margen para desarrollar prácticas que en un mismo contexto determinen resultados diferentes.

También, se ha encontrado que quienes poseen mayor estabilidad escolar y/o usan computadoras en la institución educativa obtienen mejores resultados. Aquí se vislumbra el rol que tiene la escuela sobre los logros educativos, formando parte del microsistema definido por Longás Mayayo y otros (2016).

Por último, se observa que los adolescentes que asisten a escuelas más lejanas y/o en turno mañana, al igual que quienes asisten a clases particulares y/o estudian una mayor cantidad de horas diarias, alcanzan una más alta performance. Estos elementos podrían estar dando cuenta tanto de los valores y actitudes familiares como del esfuerzo y motivación de los estudiantes. Dichas cualidades, tal como señalan Krüger y Formichella (2016), si bien pueden ser consideradas resultados escolares, también actúan como insumos en la función de producción educativa, favoreciendo la obtención de diferentes logros.

\section{Conclusión}

A lo largo de este trabajo se ha analizado en detalle la información provista por el relevamiento a hogares llevado a cabo en el marco del PDTS "Diseño de estrategias para mejorar las oportunidades educativas de la población vulnerable de Bahía Blanca a través de la ONG Red de Voluntarios". Se detectó, a través de un estudio de conglomerados, que la población de los barrios considerados puede ser agrupada según características socioeconómicas diferenciadas. Luego, se compararon indicadores 
de inclusión educativa entre estos grupos. Por último, se estudiaron subgrupos dentro de los aglomerados que presentan comportamientos y características particulares.

El análisis aquí presentado puede resultar de utilidad para el diseño de estrategias de la ONG en forma conjunta con la comunidad. En el nivel primario se destaca que, si bien la cobertura es universal, la repitencia representa un problema crucial, por lo que centrar la atención en los chicos que han repetido podría colaborar en bajar la tasa de reincidencia y evitar que la repitencia reiterada se convierta en un factor de abandono a futuro. Por su parte, en el nivel secundario, si bien nuevamente aparece como una importante dificultad la repitencia, también se visualiza el problema del abandono. Por ende, las actividades destinadas a brindar apoyo escolar (actividad principal de la ONG), por su propia esencia no abarcan a la población que ya no está asistiendo al colegio y se hace necesaria la realización de otro tipo de acciones.

En cuanto a los hacedores de políticas, los datos presentados confirman las condiciones socioeconómicas precarias presentes en estos barrios, lo cual implica que se requieren intervenciones integrales junto a una adecuada canalización de recursos en pos de mejorarlas. A su vez, en el trabajo ha quedado manifiesto que existen grupos que se encuentran en una situación aún más desfavorable que el promedio, lo cual requiere de un tratamiento prioritario. Esto, no sólo porque se vulneran distintos derechos básicos, sino porque claramente las mencionadas carencias están incidiendo en los resultados educativos de los niños y jóvenes, cerrando círculos viciosos que configuran trampas de pobreza.

Por último, cabe destacar que algunos de los hallazgos expuestos dan lugar a nuevas líneas de investigación dentro del equipo. Particularmente, a partir de la detección de variables que actúan como moderadoras y colocan a chicos pertenecientes al grupo desfavorable dentro de los que poseen los mejores resultados, resulta interesante indagar y profundizar en el rol de las mismas.

\section{Bibliografía}

Aldenderfer, M. y Blashfield, R. (1984). “Cluster Analysis”. Sage University Paper Series on Quantitative Applications in the Social Sciences No. 07-044. Sage Publications.

Andengerg, M. (1973). Cluster analysis for applications. New York: Academic Press Ed. 
Barham, B., Boadway, V., Marchand R. y Pestieau, M. (1995). "Education and the poverty trap". European Economic Review, 39, pp. 1257-1275.

Berti Ceroni, C. (2001). "Poverty traps and human capital accumulation", Economica, 68, pp. 203-219.

Bonal, X. y Zancajo, A. (2018). "Demand rationalities in contexts of poverty: Do the Poor respond to market incentives in the same way?",International Journal of Educational Development, 59, pp. 20-27.

Calinski, T. y Harabasz, J. (1974). "A dendrite method for cluster analysis." Communications in Statistics, 3(1), pp.1-27.

CREEBBA (2015). Indicadores de Actividad Económica $N^{\circ} 141$. Bahía Blanca. Disponible en: http://www.creebba.org.ar/main/index.php?op=estesp\&cual=iael $41 . h t m l \&$

Formichella, M. M. (2009). "Una explicación de las trampas de pobreza a nivel microeconómico: El círculo vicioso entre el nivel de educación y el nivel de ingresos de las familias". Revista Estudios Económicos, 26(52), pp. 49-80.

Formichella, M. M. (2010). Educación y desarrollo: análisis desde la perspectiva de la equidad educativa interna y del mercado laboral. Tesis doctoral en Economía. UNS.

Formichella, M. (2011). "¿Se debe el mayor rendimiento de las escuelas de gestión privada en la Argentina al tipo de administración?” Revista de la CEPAL, 105, pp. 151-166.

Formichella, M. y Krüger, N. (2013). "El fracaso escolar en el nivel medio argentino: ¿es menos frecuente en las escuelas de gestión privada debido a su administración?" Regional and SectoralEconomicStudies/ Estudios Económicos Regionales y Sectoriales, 13-3, pp. 127-144.

Formichella, M. y Krüger, N. (2016)."Los determinantes del rendimiento educativo en el nivel secundario argentino". Simposio Argentino sobre Economía de la Educación. IICE-PEET. Buenos Aires. 10 y 11 de agosto.

Golovanevsky, L. (2006). Vulnerabilidad y transmisión intergeneracional de la pobreza, un abordaje cuantitativo para Argentina en el siglo XXI. Buenos Aires: Universidad de Buenos Aires, Facultad de Ciencias Económica

Guevara, B. (2016). Educación secundaria en contextos de pobreza. Experiencia cotidiana y estrategias de escolarización de las familias. Cuadernos de Educación, 14(4). Disponible en: https://revistas.unc.edu.ar/index.php/Cuadernos/article/view/16934/16599

Herrero, V., Peláez, E. y González, L. (2007). "Vulnerabilidad social, rezago y deserción escolar en el Gran Córdoba (Argentina) 2001". IX Jornadas Argentinas de Estudios de Población. Huerta Grande, Córdoba.

Krüger, N. (2013). "Segregación social y desigualdad de logros educativos en Argentina”. Archivos Analíticos de Políticas Educativas, 21(86), pp. 1-30. Disponible en: http://epaa.asu.edu/ojs/article/view/1352 
Krüger, N. (2016). "Equidad educativa interna y externa: principales tendencias en Argentina durante las últimas décadas". Revista Latinoamericana de Estudios Educativos, 46(2), pp. 39-78.

Krüger, N. y Formichella, M. M. (2016). “ ¿Son las competencias no-cognitivas mediadoras en el proceso de enseñanza-aprendizaje?" Simposio Argentino sobre Economía de la Educación. IICE-PEET. Buenos Aires. 10 y 11 de agosto.

Krüger, N., Ibáñez Martín, M. M. y Formichella, M. M. "Elección escolar e inequidad educativa en el aglomerado Bahía Blanca-Cerri". En London y Pérez (comps.): Los pilares del desarrollo económico. Salud, educación y empleo. Bahía Blanca: EdiUNS.

Llach, J. (2006). El desafío de la equidad educativa. Ed. Granica.

London, S. y Autor/a, M. M. (2006). "El concepto de desarrollo de Sen y su vinculación con la Educación Economía y Sociedad". Disponible en: http://www.redalyc.org/articulo.oa?id=51001702. Consultado el 20/07/2017.

Longás Mayayo, J.; Cussó Parcerisas, I.; de Querol Duran, R. y Riera Romaní, J. (2016). "Análisis de factores de apoyo a trayectorias de éxito escolar en la enseñanza secundaria en contextos de pobreza y vulnerabilidad socia en España. Un estudio de casos múltiples". Revista de Estudios y Experiencias en Educación, 15(28), pp. 107-127.

Lopez, N. (2006). Equidad educativa y desigualdad social. Ed. IIPE-UNESCO.

Malisani, D. (2017). La política de integración urbana en Bahía Blanca a la luz del presupuesto municipal. Tesis de la Licenciatura en Economía, Universidad Nacional del Sur.

Marchionni, M., Pinto, F. y Vázquez, E. (2013). "Determinantes de la desigualdad en el desempeño educativo en la Argentina”. Reunión Anual de la Asociación Argentina de Economía Política, Rosario, 2013. Disponible en: http://www.aaep.org.ar/anales/works/works2013/marchioni_pinto.pdf

Milligan, G. y Cooper, M. (1985). "An examination of procedures for determining the number of clusters in a data set." Psychometrika, 50(2), pp. 159-179.

Prieto, M. B. (2007). "Condiciones habitacionales y calidad de vida urbana. El caso de la ciudad de Bahía Blanca”. Jornadas Argentinas de Estudios de Población. Huerta Grande, Córdoba.

Riquelme, G. y Herger, N. (2001). "El acceso y permanencia en el sistema educativo ¿quiénes son beneficiadas/os y excluidas/os?" Revista del Instituto de Investigaciones en Ciencias de la Educación, 18, pp. 3-18.

Santos, M. (2007a). "Un modelo de trampa de pobreza con capital humano y calidad de la educación", Anales de la Asociación Argentina de Economía Política.

Santos, M. (2007b). "Quality of education in Argentina: determinants and distribution using PISA 2000 test scores". Well-being and Social Policy, 3(1), pp. 69-95. 
Savova, G., Therneau, T., y Chute, C. (2006). "Cluster Stopping Rules for Word Sense Discrimination". Proceedings of the 11th Conference of the European Chapter of the Association for Computational Linguistics. Trento, Italia.

Sen, Amartya (1999). Desarrollo y Libertad. Editorial Planeta.

Sen, Amartya (2003). Teorías del Desarrollo a principios del siglo XXI. En www.comminit.com/la/teoriasdecambio/lacth $=342 . \mathrm{html}$

Tedesco, J. C. (2000). Educar en la sociedad del conocimiento. Ed. Fondo de cultura económica.

\section{Notas biográficas:}

María Marta Formichella es Investigadora Adjunta del Consejo Nacional de Investigaciones Científicas y Técnicas (CONICET) y Profesora Adjunta en el Departamento de Economía de la Universidad Nacional del Sur (UNS), Bahía Blanca, Argentina. Es Doctora en Economía por la UNS e investiga en el área de Economía de la Educación.

Filiación: Instituto de Investigaciones Económicas y Sociales del Sur, IIESS (UNS-CONICET) Departamento de Economía, Universidad Nacional del Sur (UNS), Bahía Blanca, Argentina.

Correo electrónico: mformichella@iiess-conicet.gob.ar

ORCID ID: https://orcid.org/0000-0002-2057-0938

Natalia Krüger es Investigadora Adjunta del Consejo Nacional de Investigaciones Científicas y Técnicas (CONICET) y docente en el Departamento de Economía de la Universidad Nacional del Sur (UNS), Bahía Blanca, Argentina. Es Doctora en Economía por la UNS e investiga en el área de Economía de la Educación.

Filiación: Instituto de Investigaciones Económicas y Sociales del Sur, IIESS (UNS-CONICET) Departamento de Economía, Universidad Nacional del Sur (UNS), Bahía Blanca, Argentina.

Correo electrónico: natalia.kruger@uns.edu.ar

Código ORCID: https://orcid.org/0000-0001-8401-2639

Recibido: 14-02-2018

Aceptado: 09-05-2019

(c) Licencia Creative Commons Reconocimiento (CC BY 4.0) 